Materials and Methods Between May 2015 and January 2021, E.Meshalkin National Medical Research Center and Federal Center of Brain and Neurotechnologies treated 131 AVM patients using PHIL. By January, the treatment had been completed in 70 of the patients; 59 were undergoing a staged embolization, and 2 patients had died between stages due to an AVM rupture. The data for analysis were accumulated in a prospective database.

Results The cohort for analysis included the 70 patients whose treatment was finalized. Total occlusion was achieved in 50 patients (71.4\%). Among them, 28 underwent a single-stage embolization. In 14 patients (28\%), the procedure was performed using PHIL only. A procedure, where using PHIL was combined with a single-stage microsurgical resection was performed in 2 (4\%) cases. The remaining 35 patients $(70 \%)$ underwent embolization with a combination of embolizing agents (Onyx, Squid, N-BCA) as during a single as during a multi-stage treatment. Seven patients (10\%) underwent a subtotal embolization with single-stage microsurgical resection. In 6 patients $(8.6 \%)$ subtotal embolization was followed by radiosurgery. Partial AVM occlusion with microsurgical resection and partial embolization with radiosurgical treatment were performed in $1(1.4 \%)$ and $3(4.3 \%)$ patients, respectively. Partial AVM occlusion only was performed in 3 patients $(4.3 \%)$. Intra-and postoperative complications were registered in 37 cases (53\%). Persistent neurological deficiency developed in 4 patients (5.7\%), including the two after partial embolization. The mortality rate comprised $1.4 \%$ to be 1 patient after partial AVM embolization.

Conclusion PHIL is an effective tool for treatment of brain AVMs. The agent has a high degree of initial occlusion and is safe if compared to the other liquid embolization materials. However, large-scale multicenter studies are required to determine the embolizer's safety profile and to estimate its longterm efficacy.

Disclosures K. Orlov: None. N. Strelnikov: None. V. Berestov: None. A. Somova: None.

\section{E-110 TRANSRADIAL, COAXIAL APPROACH USING SURPASS EVOLVE FOR TREATMENT OF CEREBRAL ANEURYSMS}

Z Hubbard*, B Saway, K Zilinskas, G Porto, S Al Kasab, M Sattur, A Spiotta. Medical University of South Carolina, Charleston, SC

10.1136/neurintsurg-2021-SNIS.205

Introduction The transradial approach (TRA) is being employed to a greater degree in neuroendovascular procedures. Multicenter studies examining TRA versus transfemoral approach (TFA) across all neuroendovascular procedures have demonstrated lower rates of both minor and major complications using TRA. Previous studies have described TRA for flow diversion using the Pipeline embolization device (ev3/ Chestnut Medical, Menlo Park, California) with good result. The Surpass Evolve device (SED; Stryker Neurovascular, Kalamazoo, Michigan, USA) is a new 64-wire flow diverting stent that obtained FDA approval in 2020. In this study we present a single center experience of patient who underwent TRA for placement of Surpass Evolve flow diversion for treatment of cerebral aneurysms.

Methods We performed a retrospective review of patient who underwent TRA for SED placement for treatment of aneurysm between August 2020 to February 2021. Patient demographic, procedural techniques, clinical and angiographic data were recorded.

Results 15 patients underwent SED placement for treatment of aneurysm (age 46.6, 62.5\% female). $81 \%$ of patients had hypertension, $43 \%$ had hyperlipidemia, and $75 \%$ were current or former smokers. $3(20 \%)$ patients presented with ruptured aneurysms. 7 (47\%) patients had underwent previous aneurysm treatment. 5 patients $(33 \%)$ had recurrence of their previously treatment aneurysm. 1 patient was converted to TFA after attempted TRA due to vasospasm. The following aneurysms were treated: anterior communicating artery aneurysm in 3 patients, ophthalmic artery aneurysm in 3 patients, anterior choroidal artery aneurysm in 3 patients, superior hypophyseal artery aneurysm in 3 patients, vertebral artery aneurysm in 2 patients, posterior communicating artery aneurysm in 1 patient, cervical carotid artery aneurysm in 1 patient, and a fusiform cavernous carotid aneurysm in 1 patient. The right radial artery was used in all cases. Mean radial artery diameter was $2.64 \mathrm{~mm}$. A 5/6 French slendersheath was utilized in all cases. Coaxial system was used in all cases. Mean smallest parent vessel diameter measured $3.52 \mathrm{~mm}$, and mean largest parent vessel diameter measured $4.06 \mathrm{~mm}$. Placement of the TFA flow diversion device was successful in all cases. Balloon angioplasty was used in 5 (33\%) patients to achieve wall apposition. No patients had access site complications. There were no procedural or postoperative neurological complications. 1 patient had proximal shelfing of SED. The patient required retreatment with good outcome using an atlas stent.

Conclusions TRA is a safe and feasible approach for flow diversion using the Surpass Evolve device. Rates of access site complications is favorable. Overall rate of conversion to TFA is low. Our early experience suggests frontline TRA is feasible for the utilization of Surpass Evolve flow diverting device.

Disclosures Z. Hubbard: None. B. Saway: None. K. Zilinskas: None. G. Porto: None. S. Al Kasab: None. M. Sattur: None. A. Spiotta: None.

\section{E-111 CHRONIC SUBDURAL HEMATOMA RECURRENCE DUE TO CONTRALATERAL NEOVASCULARIZATION FOLLOWING MIDDLE MENINGEAL ARTERY EMBOLIZATION}

Z Hubbard*, S Al Kasab, G Porto, M Sattur, A Spiotta. Medical University of South Carolina, Charleston, SC

10.1136/neurintsurg-2021-SNIS.206

Introduction Chronic subdural hematoma (CSDH) is one of the most commonly encountered neurosurgical diseases. Middle meningeal artery embolization (MMAE) is a technique for the management of $\mathrm{CSDH}$ that has elicited promising results. One uncommon mechanism for this recurrence of neovascularization by means of the contralateral middle meningeal artery. Despite the encouraging results of MMAE, recurrence does occur. We describe two cases of CSDH recurrence by means of contralateral middle meningeal artery neovascularization treated with contralateral MMAE.

Methods We identified two cases of recurrent subdural hematoma secondary to neovascularization following treatment with contralateral middle meningeal artery embolization.

Results Two patients initially treated with MMAE were identified with $\mathrm{CSDH}$ recurrence secondary to contralateral MMA neovascularization. There was no traumatic or coagulopathic contribution to $\mathrm{CSDH}$ recurrence. In both cases, patients 


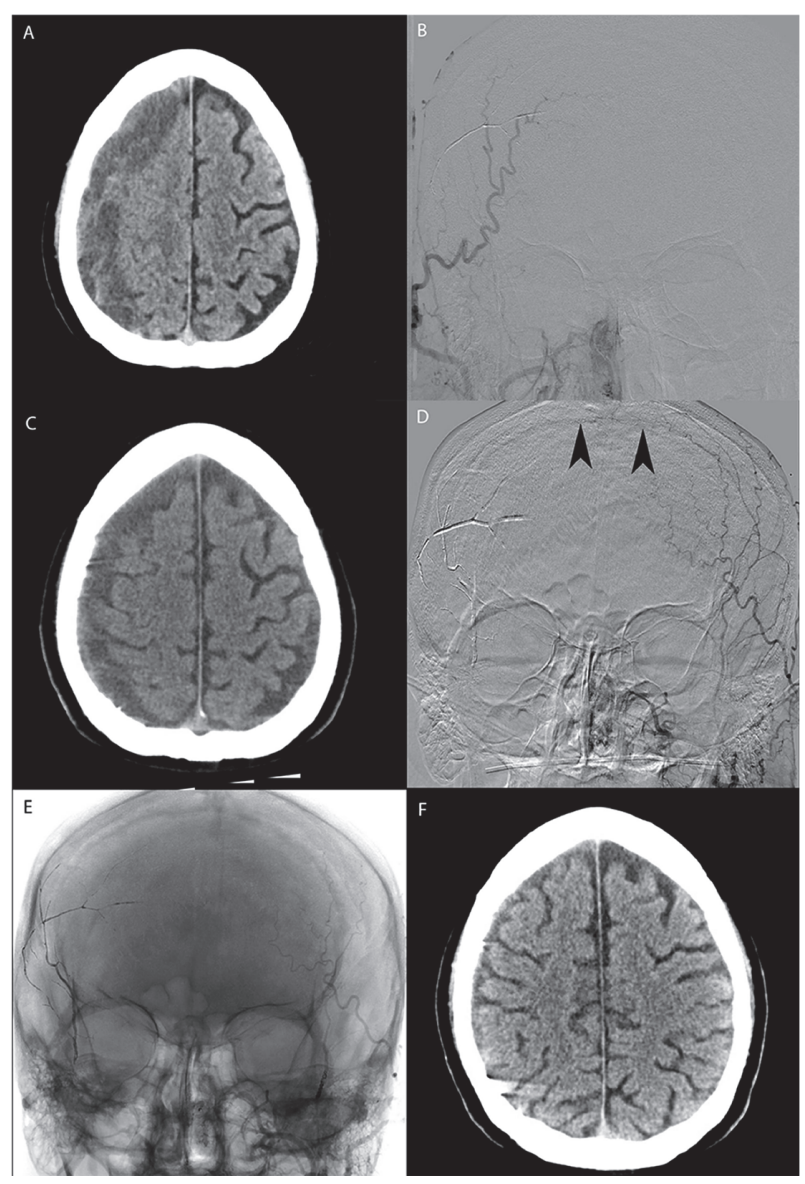

Abstract E-111 Figure 1

underwent contralateral MMAE. Both patients were neurologically intact with radiographic improvement of $\mathrm{CSDH}$ at follow up.

Conclusion Re-accumulation of SDH following MMAE by means of contralateral MMA neovascularization is a rare subtype of SDH recurrence following MMAE. Within the context of re-accumulation of SDH following MMAE catheter angiography is an important diagnostic investigation to elucidate the etiology of the recurrence. Furthermore, when angiography reveals neovascularization of the contralateral MMA, embolization of the contralateral MMA achieves good clinical and radiographic result.

Disclosures Z. Hubbard: None. S. Al Kasab: None. G. Porto: None. M. Sattur: None. A. Spiotta: None.

\section{E-112 INFANTILE TRAUMATIC PERICALLOSAL ANEURYSM: A CASE REPORT}

Z Hubbard*, G Porto, S Al Kasab, M Sattur, A Spiotta. Medical University of South Carolina, Charleston, SC

\subsection{6/neurintsurg-2021-SNIS.207}

Background and Importance Traumatic aneurysms are a rare sequelae of non-accidental head trauma in infants. The rate of non-accidental trauma in the pediatric population is increasing, therefore traumatic aneurysms are an important consideration in the evaluation of pediatric patients with abusive head trauma. We report a 4-week-old infant who suffered a traumatic aneurysm arising from the pericallosal artery.

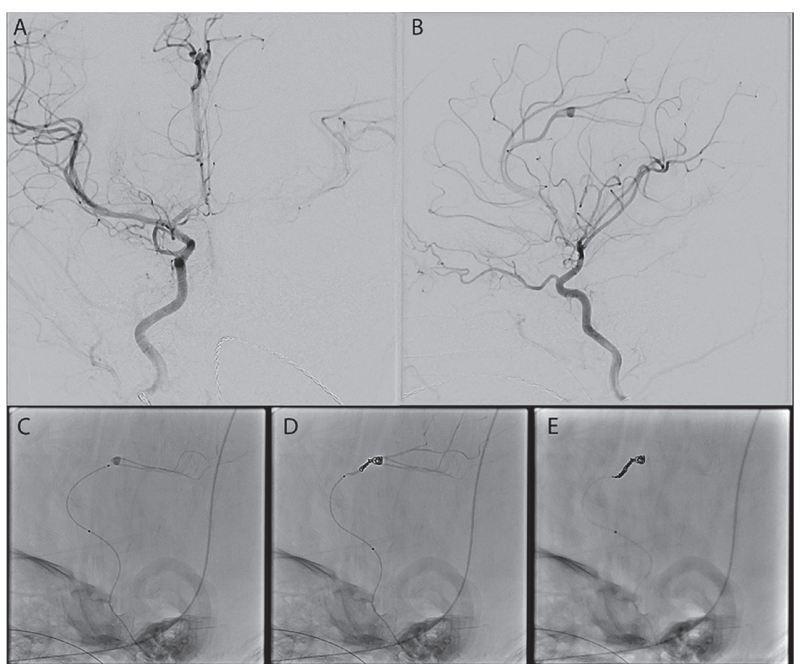

Abstract E-112 Figure 1

Case Presentation A 24 day old infant with no significant past medical or birth history presented with twitching and poor oral intake for one day prior to presentation. The patient was found to have bilateral SDH, multifocal contusions, and traumatic subarachnoid hemorrhage. NAT workup was remarkable for repeated and prolonged period of abuse. MRA revealed a pericallosal traumatic aneurysm that was treated by means of onyx/coil embolization.

Conclusion Traumatic intracranial aneurysms are a rare, but serious sequelae of pediatric abusive trauma. The authors present a case of a pericallosal traumatic aneurysm as a result of abuse that was treated with coil/onyx embolization of the aneurysm and parent artery occlusion. Rates of pediatric abuse are increasing, and as such traumatic intracranial aneurysms will remain a challenging entity to identify and treat. Despite new methods for management of traumatic aneurysms, prognosis remains poor due to the diffuse injury often involved in these patients.

Disclosures Z. Hubbard: None. G. Porto: None. S. Al Kasab: None. M. Sattur: None. A. Spiotta: None.

\section{E-113 ENDOVASCULAR RECURRENCE OF CIRCLE OF WILLIS ANEURYSMS: RE-ACCESS FOLLOWING FLOW DIVERSION}

${ }^{1} \mathrm{~J}$ Steele* ${ }^{2}{ }^{\mathrm{T}}$ Payner, ${ }^{2} \mathrm{C}$ Kulwin, ${ }^{2} \mathrm{D}$ Sahlein. ${ }^{1}$ Michigan Head and Spine Institute, southfield, MI: ${ }^{2}$ Goodman Campbell Brain and Spine, Carmel, IN

\subsection{6/neurintsurg-2021-SNIS.208}

Introduction Clinically significant aneurysm recurrence after surgical clipping, regardless of size, is low at 1\%. Clip reconstruction of large and giant aneurysms, however, have a higher rate of retreatment of $8 \%$ and consequent patient morbidity (12\%) and mortality (7\%). Endovascular flow diversion has decreased the treatment morbidity and mortality $(7 \%, 4 \%$ respectively) of large and giant aneurysms significantly with 5 year residual aneurysm rate of $5 \%$. Further flow diversion of residual aneurysm regrowth has the potential for occluding parent vessel branches and increasing patient morbidity. The authors present a case series of endovascular aneurysm recurrence following flow diversion treated by using the Circle of Willis to directly access and treat the aneurysm recurrence from the adjacent circulation. 